\title{
AN INDECOMPOSABLE LIMIT SUM
}

BY N. E. RUTT

It is the object of this paper to investigate a certain simple monotone sequence of continua. The theorem of the paper states conditions under which the limit sum of the sequence is indecomposable. The precise formulation and proof of the theorem will be undertaken after the following lemma is established.

Lemma. Let $K$ be a plane bounded indecomposable continuum and $L$ a plane bounded continuum such that $K \cdot L \neq 0$, and that $c(L) *$ includes a particular component $\lambda$ containing the component $\delta$ of $c(L+K)$ with the following properties:

(a) the set $L$ contains two distinct points, $a$ and $c$, connected through $\delta$ by the arc $B$ which divides $\delta$ into $\delta_{i}$ and $\delta_{e}$, and $\lambda$ into $\lambda_{i}$ and $\lambda_{e}$;

(b) both $\lambda_{i}$ and $\lambda_{e}$ contain points of $K$.

Then each component of $c(K+L)$ has as its boundary a proper subset of $K+L$.

The assumption that $c(K+L)$ has a component $\gamma$ with boundary $\Gamma$ such that $\Gamma \supset(K+L)$ will be shown contradictory. Let the boundaries of $\delta_{i}, \delta_{e}, \lambda_{i}, \lambda_{e}$ be respectively $\Delta_{i}, \Delta_{e}, \Lambda_{i}$, and $\Lambda_{e}$. Suppose that $\delta$ is unbounded and also $\delta_{e}$ and $\lambda_{e}$, so that $\delta_{i}$ and $\lambda_{i}$ will necessarily be bounded. Evidently $\lambda_{i} \supset \delta_{i}$ and $\lambda_{e} \supset \delta_{e}$. Consider first the case in which $L$ is irreducible between $a$ and $c$.

Both $\Lambda_{i}$ and $\Lambda_{e}$ contain $L$. For $\Lambda_{i} \subset L+B$ and $\Lambda_{e} \subset L+B$; so, since $B$ is an arc with $L \cdot(B) \dagger=0, \Lambda_{i} \cdot L$ and $\Lambda_{e} \cdot L$ are continua containing $a+c$. If either of these is not identical with $L$, then $L$ is reducible between $a$ and $c$. The domains $\delta$ and $\gamma$ are, moreover, identical, for both $\lambda_{i}$ and $\lambda_{e}$ contain points of $K$, therefore points of $\Gamma$, and therefore points of $\gamma$. There is thus an arc $X$ in $\gamma$ such that $X \cdot \lambda_{i} \neq 0$ and $X \cdot \lambda_{e} \neq 0$, and since $X \cdot L=0$, then $(B) \cdot X \neq 0$. This implies $X \cdot \delta \neq 0$, accordingly $\gamma \cdot \delta \neq 0$; and as both $\gamma$ and $\delta$ are components of $c(K+L)$, then $\gamma=\delta$, and $\lambda+L \supset \Gamma \supset K$.

Let $K_{i}$ be the sum of $K \cdot \lambda_{i}$ and of all the components of $L \cdot K$

* If $X$ is a point set then $c(X)$ is the complement of $X$.

$\dagger$ If $X$ is an arc then $(X)$ is $X$ with ends omitted. 
containing limit points of $K \cdot \lambda_{i}$; and let $K_{e}$ be a similar set constructed from $\lambda_{e}$. Each of these is a closed subset of $K$; and $K_{i}+K_{e} \supset K \cdot\left(\lambda_{i}+\lambda_{e}\right) \supset K \cdot \lambda$, so that $K_{. i}+K_{e} \supset \overline{K \cdot \lambda}$. If $K_{i}+K_{e}$ $\$ K \cdot L$, then $K \cdot L$ includes a component $M$ such that $\overline{K \cdot \lambda} \cdot M=0$ and $M$ may be enclosed in a simple closed curve $C$, not intersecting $K \cdot L$, and excluding $\overline{K \cdot \lambda}$. But $C \cdot(\overline{K \cdot \lambda}+K \cdot L)=0$ implies $C \cdot K=0$, because $\lambda+L \supset K$, so $C$ separates $K$ without intersecting it. Accordingly $K_{i}+K_{e}=K \cdot \lambda+K \cdot L=K$. But now if both $K_{i}$ and $K_{e}$ were continua, as they are proper subsets of $K$, the set $K=K_{i}+K_{e}$ would be decomposable. Thus either $K_{i}$ or $K_{e}$ is disconnected.

Let $K_{i}$ be disconnected, that is, let $K_{i}=K_{a}+K_{c}$ where $K_{a}=\bar{K}_{a}, K_{c}=\bar{K}_{c}$, and $K_{a} \cdot K_{c}=0$. As $\lambda_{i} \cdot K$ is contained by $\Delta_{i}$, the components of $K \cdot \lambda_{i}$ are an orderable collection and elements of this collection accessible from $\delta_{i}$ must belong to both $K_{a}$ and $K_{c}$. Thus there is a pair of $\operatorname{arcs} B_{a}$ and $B_{c}$ where $\delta_{i} \supset\left\{\left(B_{a}\right)+\left(B_{c}\right)\right\}, \quad B_{a} \cdot K_{a} \cdot \lambda_{i} \neq 0, \quad B_{c} \cdot K_{c} \cdot \lambda_{i} \neq 0, \quad B_{a} \cdot(B) \neq 0$, $B_{c} \cdot(B) \neq 0$, and $B_{a} \cdot B_{c}=0$. Now $B_{a}+B+B_{c}$ contains an arc $D$, such that $D \supset B_{a}+B_{c}, D \cdot B$ is a subarc of $(B),(D) \subset \delta$, and $\delta-(D)$ is a pair of domains $\delta_{u}$ and $\delta_{b}$. Let the notation be so chosen that $\delta_{b} \subset \delta_{i}$ and $\delta_{u} \supset \delta_{e}$, the relationships being clear from the construction; and let the boundaries of $\delta_{u}$ and $\delta_{b}$ be $\Delta_{u}$ and $\Delta_{b}$. Note that $\delta_{b} \cdot(a+c)=0$.

Now $\Delta_{b} \cdot(K+L) \nsubseteq K$. For $\Delta_{b} \supset D$ and consequently $\Delta_{b} \cdot K_{a} \neq 0$ and $\Delta_{b} \cdot K_{c} \neq 0$. But $\Delta_{b} \cdot(K+L)$ is a continuum because $\Delta_{b} \cdot c(K+L)=(D)$. As $K_{a} \cdot K_{c}=0,{\overline{\Delta_{b} \cdot K_{a}}}_{a} \cdot{\overline{\Delta_{b} \cdot K_{c}}}_{c}=0$; that is $\Delta_{b} \cdot(K+L) \Phi \Delta_{b} \cdot\left(K_{a}+K_{c}\right)$. Therefore $\Delta_{b} \cdot L \cdot c\left(K_{a}+K_{c}\right)=L_{k} \neq 0$. Moreover $L_{k}$ includes a component $L_{b}$ such that $\overline{L_{b}} \cdot K_{a} \neq 0$ and $\overline{L_{b}} \cdot K_{c} \neq 0$, for otherwise it must follow again that $\Delta_{b}-(D)$ is disconnected. Now $K_{e} \ngtr L_{b}$, as otherwise there must be a component of $K \cdot L$ containing $L_{b}$ and thus contained in both $K_{a}$ and $K_{c}$. Thus $K_{i}+K_{e} \ngtr L_{b}$ and $L \cdot c(K) \supset h$, a point distinct from $a$ and from $c$, and in $\Delta_{b}$.

Let $S$ be a circle with center $h$ and radius such that $c e(S)^{*}$ is a set with no points in $K+D+B$, and such that in $e(S) \cdot \delta_{e}$ there is a ray $R$ with end on $(B)$. As $L$ is irreducible between $a$ and $c, L \cdot c i(S)$ consists of sets $L_{a}^{\prime \prime}$ and $L_{c}^{\prime \prime}$ mutually separated

* If $S$ is a simple closed curve, its interior is $i(S)$ and its exterior is $e(S)$. Accordingly $c i(S)=S+e(S)$ and $c e(S)=S+i(S)$. 
between $a$ and $c$. Let $L_{a}$ and $L_{c}$ be the components of these containing $a$ and $c$. Evidently $S \cdot L_{a} \neq 0$ and $S \cdot L_{c} \neq 0$. Let $F$ be an arc such that $F \cdot S \neq 0,(F) \subset e(S),(F) \subset \delta_{b}$, and $F \cdot(D \cdot B) \neq 0$. Let $E$ be an arc such that $E \cdot S \neq 0,(E) \subset e(S),(E)+E \cdot S \subset \lambda_{e}$, and $E \cdot B=F \cdot B$. Let $G$ be an arc such that $(G) \subset i(S)$ and $G \cdot S$ $=(E+F) \cdot S$. The set $E+F+G$ is a simple closed curve $J$, intersecting $L$ only in $i(S)$.

The set $L_{a}+c e(S)+F+B$ bounds a bounded domain $\phi_{a}$ containing points of $K \cdot c(L) \cdot \lambda_{i}$; and $L_{c}+c e(S)+F+B$ bounds a similar domain $\phi_{c}$. For $L_{a}+c e(S)$ is obviously a continuum and $B+F$ contains a cut $F_{a}$ of its complement. Of the two components of $c\left(F_{a}+L_{a}+c e(S)\right)$ which are bounded in part by $\left(F_{a}\right)$ let $\phi_{a}$ be the bounded one. The boundary of $\phi_{a}$ contains either $B \cdot B_{a}$ or $B \cdot B_{c}$ (suppose the former), but not of course both. Thus by elementary reasoning from the constructions used, it appears that $\phi_{a} \supset B_{a} \cdot c(B)$ and thus contains a component of $K_{a} \cdot \lambda_{i}$. In a similar way $\phi_{c}$ may be proved to contain a component of $K_{c} \cdot \lambda_{i}$. Moreover $\phi_{a} \cdot \phi_{c}=0$ and if $i(J) \supset \phi_{a}$ then $e(J) \supset \phi_{c}$ and vice versa. To be explicit, assume that $i(J) \supset \phi_{a}$.

That the domain $\lambda_{e}$ contains an uncountably infinite number of components of $\lambda_{e} \cdot K$ each of which has a disconnected set of limit points in $H$ will now be shown. Since $i(J) \supset \phi_{a}$ and $e(J) \supset \phi_{c}$, both $i(J)$ and $e(J)$ contain points of $K \cdot c(H)$ and thus contain points of every composant of $K$. Let $\left[Q_{\alpha}\right]$ be a collection of subcontinua of $K$, one and only one in each composant of $K$, and each one having both a point in $\phi_{a}$ and a point in $\phi_{c}$. The elements of $\left[Q_{\alpha}\right]$ are uncountable and mutually exclusive. Any one, $Q_{o}$, of $\left[Q_{\alpha}\right]$ has a point in $\Phi_{a}$ and one in $\Phi_{c}$, where $\Phi_{a}$ and $\Phi_{c}$ are the boundaries respectively of $\phi_{a}$ and $\phi_{c}$; so $Q_{v} \cdot \Phi_{a}=Q_{v} \cdot\left(L_{a}+S+F_{a}\right)=Q_{v} \cdot L_{a} \neq 0$, and also $Q_{v} \cdot L_{c} \neq 0$. It appears indeed that $Q_{\sigma}$ has a point in each of the mutually separated closed sets $L \cdot e(J) \cdot c i(S)$ and $L \cdot i(J) \cdot c i(S)$, and as these two sets contain $L \cdot K$ they contain $L \cdot Q_{0}$. In consequence $Q_{o} \cdot c(L)$ has a component $G_{o}$ with a limit point in $L \cdot i(J)$ and a limit point in $L \cdot e(J)$. As $L \cdot i(J)$ and $L \cdot e(J)$ are mutually separated, the limit set of $G_{0}$ is disconnected since it includes no point of $J$; and also $G_{g} \cdot J=G_{g} \cdot(E+F+G)=G_{g} \cdot E \neq 0$, so $G_{g} \cdot \lambda_{e} \neq 0$ and therefore $\lambda_{e} \supset G_{g}$. Regard now the components $\left[K_{\alpha}\right]$ of $\lambda_{\theta} \cdot K$ which contain the members of $\left[G_{\alpha}\right]$. Each of these has limit points in both $i(J)$ and $e(J)$ and none in $J$, and so 
has a disconnected limit set in $L$. No two of these are identical, for no one, such as $K_{\theta}$, of them has $\bar{K}_{g}=K$ as it contains none of the points of $K$ in $\phi_{a}+\phi_{c}$, and if $K_{o}$ were to contain two of $\left[G_{\alpha}\right]$ then $K_{o}$ would be a proper subcontinuum of $K$ containing points of two different composants of $K$. Thus the collection $\left[K_{\alpha}\right]$ is one of the sort required.

Each set $K_{v}+L$ is therefore a subcontinuum of $K+L$ separating the plane, a bounded component of its complement being $\delta_{g}$. No pair of elements, $\delta_{p}$ and $\delta_{q}$, of $\left[\delta_{\alpha}\right]$ can have a point in common unless one contains the other, for $K_{p} \cdot K_{q}=0$. Moreover if $\delta_{p} \supset \delta_{q}$ then $\delta_{p} \supset K_{q}$, and so $\delta_{p} \supset \gamma$, a contradiction as $\gamma \supset \delta$ was unbounded. Thus $\left[\delta_{\alpha}\right]$ is an uncountable collection of mutually exclusive domains in the plane, another contradiction establishing at last the lemma for this case.

None of the undiscussed suppositions made above requires any more justification than a suitable inversion of the plane except the assumption that $L$ is irreducible between $a$ and $c$. But if $L$ is not irreducible between $a$ and $c$, then it contains a subcontinuum $W$ which is irreducible between $a$ and $c$. By examining $K$ and $W$ it may be seen that the hypotheses of the lemma are fulfilled, so that the set $c(K+W)$ has no component with boundary $K+W$. Neither then does the less inclusive set $c(K+H)$.

Theorem. If $\left[D_{i}\right]$ is a simple infinite sequence of plane point sets such that, for each positive integer $i, D_{i}$ is indecomposable and $D_{i} \subset D_{i+1}$, and such that the set $\overline{\sum_{1}^{\infty} D_{i}}$ is a plane bounded continuum $\Gamma$ which is the frontier of $\gamma$, a component of its complement, then $\Gamma$ is also indecomposable.

The theorem is obvious if no more than a finite number of $\left[D_{i}\right]$ are distinct, as then $\Gamma$ is identical with one of $\left[D_{i}\right]$. Assume accordingly that all of $\left[D_{i}\right]$ are different, other possible cases being not significant. Let $\sum_{1}^{\infty} D_{i}=D_{m}$ and $\Gamma-D_{m}=D_{n}$. Every point of $D_{n}$ is a limit point of $D_{m}$, for $\Gamma=\overline{\sum_{1}^{\infty} D_{i}}=\overline{D_{m}}$ so $\overline{D_{m}} \supset D_{n}$. Every subcontinuum of $D_{n}$ is a continuum of condensation of $\Gamma$, because when $D_{n}$ contains the continuum $K$, then $\overline{D_{m}} \supset D_{n} \supset K$ implies $\overline{\Gamma-K} \supset \overline{\Gamma-D_{n}} \supset \overline{D_{m}} \supset K$. Moreover let $d$ be a point of $D_{m}$. Now $D_{j+1} \supset D_{j}$ while $D_{j+1}-D_{j} \neq 0$, so $D_{j}$ belongs to a single composant of $D_{j+1}$. Thus $\overline{D_{j+1}-D_{j}} \supset D_{j}$; that 
is ${\overline{\Gamma-D_{j}}}_{j} \supset \overline{D_{j+1}-D_{j}} \supset D_{j}$, or $D_{j}$ is a closed set nowhere dense in the closed set $\Gamma$. But this being true for any value of $j$ $(j=1,2,3, \cdots)$ then $\sum_{1}^{\infty} D_{j}=D_{m}$ must be a set of the first category in the closed set $\Gamma$. Thus $D_{n}$ is a set of the second category in $\Gamma$ everywhere dense in the set $\Gamma$. That is, $\overline{D_{n}} \supset D_{m}$ so $\overline{D_{n}} \supset d$. Consequently every subcontinuum of $D_{m}$ is a continuum of condensation of $\Gamma$, for when $D_{m} \supset K$, then $\overline{D_{n}}=\Gamma$ implies that $\overline{\Gamma-K} \supset \overline{\Gamma-D_{m}} \supset \overline{D_{n}} \supset K$. Henceforth consider $\gamma$ unbounded.

The argument will be completed by showing that every proper subcontinuum of $\Gamma$ is a continuum of condensation of $\Gamma$. Let $K$ be such a continuum and, as the cases $D_{m} \supset K$ and $D_{n} \supset K$ have already been dealt with, suppose that $K \cdot D_{m} \neq 0$ and $K \cdot D_{n} \neq 0$. Clearly $K \triangleright D_{m}$ for $K \supset D_{m}$ would imply $K=\Gamma$. There must be some element of $\left[D_{i}\right]$ contained in part but not entirely by $K$. Let $D_{k}$ be such an element. Thus, if $i>k$, the element $D_{i}$ can not be a subset of $K$, for $K \supset D_{i}$ implies $K \supset D_{i} \supset D_{k}$.

Suppose that the set $\overline{D_{k} \cdot c(K)}=\Gamma_{k}$ is not connected. As $D_{k}+K \subset \Gamma, c\left(D_{k}+K\right) \supset c(\Gamma) \supset \gamma$. Thus there is a connected domain $\gamma_{k}$ complementary to $D_{k}+K$ such that $\gamma_{k} \supset \gamma$. As $\bar{\gamma}_{k} \supset \bar{\gamma} \supset \Gamma$ then $\bar{\gamma}_{k} \supset D_{k}+K$. But $\bar{\gamma}_{k}=\gamma_{k}+B_{k}$, where $B_{k}$ is the boundary of $\gamma_{k}$, and so $\bar{\gamma}_{k} \supset D_{k}+K$ implies $B_{k} \supset D_{k}+K$. Let $G$ be a component of $\Gamma_{k}$ containing the end of a ray $R_{g}$ contained except for its end in $\gamma_{k}$. As every component of $\Gamma_{k}$ consists of limit points of $\gamma_{k}$, and $\Gamma_{k}$ is not connected, there is another component $H$ of $\Gamma_{k}$ containing the end of another ray $R_{h},\left(R_{g} \cdot R_{h}=0\right)$, which is except for its end contained in $\gamma_{k}$.

Now there exists a simple closed curve $C$ such that $i(C) \supset G$, $C \cdot \Gamma_{k}=0, e(C) \supset H+R_{h}$, and $C \cdot R_{o}$ is a single point. Upon tracing $C$ in opposite directions from $C \cdot R_{g}$, first points of $D_{k}+K$ are clearly encountered. Let the subarc of $C$ thus identified be $B$. But $(B) \cdot\left(D_{k}+K\right)=0$ by selection, and $B \cdot\left(D_{k}+K\right) \subset B \cdot\left(\Gamma_{k}+K\right)$ $\subset C \cdot \Gamma_{k}+B \cdot K \subset B \cdot K \subset K$, so $B$ is a cut of the unbounded complementary domain $\gamma_{d}$ of $K$. Thus $\gamma_{d}-(B)$ consists of two domains, a bounded one $\gamma_{b}$ and an unbounded one $\gamma_{u}$. As $R_{h} \cdot(B+K) \subset R_{h} \cdot(C+K) \subset R_{h} \cdot C+R_{h} \cdot K=0$, then $\gamma_{u} \supset R_{h}$, and thus $\gamma_{u} \cdot D_{k} \neq 0$, for indeed $\gamma_{u} \supset H$. Upon considering $\gamma_{b}$ it may be seen with reasonable ease that the single point $B \cdot R_{g}$ separates $R_{o}$ into two parts, the unbounded one of which is a subset of $\gamma_{u}$ whereas the bounded one is a subset of $\gamma_{b}$. As the end of 
the bounded part is in $G$, then $\gamma_{b}$ also contains points of $\Gamma_{k}$ as it contains $G$. These facts make it clear also that the ends of $B$ are distinct, for if identical they would coincide with a cut point of the continuum $D_{k}$, although $D_{k}$ is indecomposable.

A contradiction of the lemma now appears, for this is the situation: the plane continuum $D_{k}$ is indecomposable and bounded and $K$ is a bounded continuum such that $K \cdot D_{k} \neq 0$, the set $K$ contains two distinct points connected in $c\left(D_{k}+K\right)$ by an arc $B$ having only its ends in common with $D_{k}+K$ and separating the component of $c(K)$ which contains it into two domains $\gamma_{b}$ and $\gamma_{u}$, both $\gamma_{b}$ and $\gamma_{u}$ contain points of $D_{k}$, and there is a component of $c\left(D_{k}+K\right)$ whose boundary is identical with $D_{k}+K$. As this is ridiculous, the set $\Gamma_{k}$ is connected as was to be proved.

But if $K$ is not a continuum of condensation of $\Gamma$, there exists. a point $s$ of $K$ and a circle $S$ such that $i(S) \supset s$ and $e(S) \supset \overline{\Gamma-K}$. But $\overline{D_{m}}=\Gamma$ so $i(S) \cdot D_{m} \neq 0$; that is, there exists a subscript $q$ such that $i(S) \cdot D_{q} \neq 0$. For any subscript $j>q$, then $i(S) \cdot D_{j} \neq 0$ as $i(S) \cdot D_{j} \supset i(S) \cdot D_{q} \neq 0$. Let $r$ be a natural number greater than $k$ and greater than $q$. Then $\overline{D_{r} \cdot c(K)}=\Gamma_{r}$ is non-vacuous and connected as has been seen already. But $\Gamma_{r} \cdot i(S) \subset \overline{\Gamma-K} \cdot i(S)=0$, so $\Gamma_{r}$ fails to contain any point of the non-vacuous set $i(S) \cdot D_{r}$. Therefore $\Gamma_{r}$ is a proper subcontinuum of $D_{r}$, and must accordingly belong to a single composant $D_{r}{ }^{a}$ of $D_{r}$. As $D_{r} \cdot c(K) \neq 0$ by supposition, there are points of $D_{r}$ not in $K$. But let $D_{r}^{b}$ be a second composant of $D_{r}$. As $D_{r}^{a} \supset \Gamma_{r}$, then $D_{r}^{b} \subset K$. Accordingly $\overline{D_{r}^{b}} \subset K$, and as $\overline{D_{r}^{b}}=D_{r}$, finally $D_{r} \subset K$, a contradiction.

As the contradiction is now general, the theorem is proved.

Louisiana State University 